\title{
Striatal modulation of BDNF expression using microRNA124a-expressing lentiviral vectors impairs ethanol-induced conditioned-place preference and voluntary alcohol consumption
}

\author{
Amine Bahi ${ }^{1}$ and Jean-Luc Dreyer ${ }^{2}$ \\ ${ }^{1}$ Department of Anatomy, Tawam Medical Campus, CMHS, United Arab Emirates University, Al Ain, UAE \\ ${ }^{2}$ Division of Biochemistry, Department of Biology, University of Fribourg, Fribourg, Switzerland
}

Keywords: BDNF, conditioned place preference, lentiviral vector, microRNA, miR124a, siRNA

\begin{abstract}
Alcohol abuse is a major health, economic and social concern in modern societies, but the exact molecular mechanisms underlying ethanol addiction remain elusive. Recent findings show that small non-coding microRNA (miRNA) signaling contributes to complex behavioral disorders including drug addiction. However, the role of miRNAs in ethanol-induced conditioned-place preference (CPP) and voluntary alcohol consumption has not yet been directly addressed. Here, we assessed the expression profile of miR124a in the dorsal striatum of rats upon ethanol intake. The results show that miR124a was downregulated in the dorso-lateral striatum (DLS) following alcohol drinking. Then, we identified brain-derived neurotrophic factor (BDNF) as a direct target of miR124a. In fact, BDNF mRNA was upregulated following ethanol drinking. We used lentiviral vector (LV) gene transfer technology to further address the role of miR124a and its direct target BDNF in ethanol-induced CPP and alcohol consumption. Results reveal that stereotaxic injection of LV-miR124a in the DLS enhances ethanol-induced CPP as well as voluntary alcohol consumption in a two-bottle choice drinking paradigm. Moreover, miR124a-silencer (LV-siR124a) as well as LV-BDNF infusion in the DLS attenuates ethanol-induced CPP as well as voluntary alcohol consumption. Importantly, LV-miR124a, LV-siR124a and LV-BDNF have no effect on saccharin and quinine intake. Our findings indicate that striatal miR124a and BDNF signaling have crucial roles in alcohol consumption and ethanol conditioned reward.
\end{abstract}

\section{Introduction}

Ethanol abuse is a major health economic and social concern in modern societies. Substance abuse, including alcohol, is characterised by compulsive drug taking and seeking, and the consumption of substances of abuse converges on a shared pathway within the limbic system that mediates motivated behaviors (Nestler \& Carlezon, 2006; Stuber et al., 2010). The dorso-lateral striatum (DLS) is involved in the development of habits, including maladaptive persistent habits (Yin et al., 2004, 2006; Yin \& Knowlton, 2006; Tricomi et al., 2009), and is involved in advanced stages of addiction, when drug use progresses towards a compulsive, habitual pathology and behaviors directed mostly at drug seeking (Gerdeman et al., 2003). The transition from voluntary drug use to more compulsive and habitual modes of drug-seeking behavior represents a transition from prefrontal cortical to striatal control over-responding, and from ventral to more dorsal striatal subregions (Everitt \& Robbins, 2005; Everitt et al., 2008; Everitt \& Robbins, 2013). An increase in dopaminergic transmission is observed to occur either by direct action of

Correspondence: Dr A. Bahi, as above.

E-mail: amine.bahi@uaeu.ac.ae drugs on dopaminergic neurons (cocaine, nicotine) or indirectly by inhibition of $\gamma$-aminobutyric acid-ergic interneurons in the ventral tegmental area (alcohol, opiates; Nestler \& Carlezon, 2006). As for most substances of abuse, ethanol abuse is typically a multigenetic brain disorder, implying combined changes of expression of several hundred genes, producing its rewarding effects through an interaction with a large array of brain pathways, and leading to persistent alterations (neuroplastic, structural and functional) in related brain centers. Neuroadaptations underlying behavioral sensitisation result in long-lasting functional changes in these brain circuits, increasing compulsive patterns of ethanol seeking and craving (Simerly, 2006). Epigenetic modifications and neuroadaptations imply gene expression changes of a large array of genes whose control is not yet established, as the exact molecular mechanisms underlying ethanol addiction are poorly understood.

Recently, however, the roles of some microRNAs (miRNAs) in mammalian midbrain dopaminergic neurons have been identified. miRNAs are small non-protein-coding RNA transcripts that can regulate the expression of messenger RNAs that code for proteins. Because of their highly pleiotropic nature, each miRNA has the potential to regulate hundreds or even thousands of protein-coding RNA transcripts (Thomson et al., 2006; Filipowicz et al., 2008; 
Jonkman \& Kenny, 2013). miRNAs therefore are important epigenetic regulators, and control important signaling patterns (Cao et al., 2006; Franke et al., 2012), contributing to various disease processes (Kuss \& Chen, 2008; Silber et al., 2009; Sato et al., 2011). miRNAs act as key spatiotemporal regulators in post-transcriptional control of gene expression during dendritic morphogenesis and synapse development, controlling the expression of hundreds of genes involved in neuroplasticity and in the function of synapses (Silber et al., 2009; Dreyer, 2010; Sato et al., 2011; Sun et al., 2012). Moreover, miRNAs have been found to relate to addictive behaviors (Kim et al., 2007; Dreyer, 2010; Impey et al., 2010). miRNAs in the dorsal striatum control the escalation of drug intake in rats (Jonkman \& Kenny, 2013), highlighting the central role for miRNAs in drug-induced neuroplasticity in brain reward systems that drive the emergence of compulsive-like drug use in animals. For example, expression changes of miR124a in the brain reward pathway strongly affect behavior towards drugs of abuse, for example cocaine (Chandrasekar \& Dreyer, 2011), and miR124a regulates cocaine-induced expression plasticity (Chandrasekar \& Dreyer, 2009). miR124a is strongly expressed in the brain and regulates adult neurogenesis (Cao et al., 2007; Cheng et al., 2009; Kawahara et al., 2012), and promotes neuronal differentiation by triggering brain-specific alternative pre-mRNA splicing (Makeyev et al., 2007). In addition, it is involved in several brain pathologies, including aging and Alzheimer's disease (Fang et al., 2012; Qi et al., 2012). miR124a targets and regulates the expression of both the mRNA of the anti-neural function protein SCP1 (small C-terminal domain phosphatase 1; Visvanathan et al., 2007) and of the Sry-related box Sox9 transcription factor whose overexpression abolishes neuronal differentiation (Cheng et al., 2009). Besides these important functions, miR124 also plays putative roles in hepatocellular carcinoma (Zheng et al., 2012), gastric cancer (Xia et al., 2012b), and in brain tumor differentiation and progression (Silber et al., 2008; Xia et al., 2012a). Finally, miR124a in the dopaminergic midbrain pathway also strongly affects the expression of several neurotransmitters, mainly brain-derived neurotrophic factor (BDNF; Chandrasekar \& Dreyer, 2009,2011 ), which in turn is involved in the survival and function of midbrain dopaminergic neurons and also in drug reward and relapse (Corominas et al., 2007; Briand \& Blendy, 2010; Ghitza et al., 2010). Furthermore, alcohol-induced expression changes of some miRNAs induce changes at protein levels, disrupt tight junctions, and increase cell permeability and drastically affect cell signaling (Kim et al., 2007; Dreyer, 2010; Hollander et al., 2010), thus providing evidence of the control of mRNA expression by miRNAs in alcohol abuse.

Our previous studies have shown a crucial function of BDNF, through its receptor tropomyosin-related kinase B (TrkB), in the enhancement of locomotor activity, behavioral sensitisation, cocaine-induced conditioned-place preference (CPP) acquisition as well as extinguished cocaine-induced CPP reinstatement following cocaine priming (Bahi et al., 2008). Human BDNF expression is controlled by complex mechanisms, and its transcription is regulated by multiple promoters driving the expression of different coding transcripts (Pruunsild et al., 2007; Caputo et al., 2011). Genes bearing multiple binding sites for transcription factors show higher probabilities to be targeted by miRNAs and to harbor more miRNA-binding sites on average (Cui et al., 2007; Caputo et al., 2011). Indeed, BDNF expression is regulated by a group of miRNAs, and to date some of these sites have been experimentally validated [miR-1/206 (Lewis et al., 2003); miR-30a, miR-30a-5p and miR195 (Mellios et al., 2008); miR-124 and let-7d (Chandrasekar \&
Dreyer, 2009); miR-15a (Friedman et al., 2009); and miR-210 (Fasanaro et al., 2009)].

In the present study we further evaluate the role of BDNF and miR124a in alcohol abuse, using viral manipulation of local gene expression of either miR124a or BDNF as described in our previous studies (Karpova et al., 2011; Bahi \& Dreyer, 2012), and we show that striatal modulation of both miR124a and BDNF expression impairs ethanol-induced $\mathrm{CPP}$ and voluntary alcohol intake in rats.

\section{Materials and methods}

\section{Animals}

Adult male Wistar rats were group-housed (five per cage) at room temperature (approximately $22^{\circ} \mathrm{C}$ ) with a $12: 12 \mathrm{~h}$ light : dark cycle (light on at 06:00 h), and allowed to adapt to this environment for a period of 7 days before the experiments began. Bedding was produced locally and autoclaved before use, and rats had free access to tap water and standard rodent chow diet obtained from the National Feed and Flour Production and Marketing Company LLC (Abu Dhabi, UAE). All animal care and use were in accordance with the National Institutes of Health 'Guide for the Care and Use of Laboratory Animals'. The procedures were approved by the institutional Animal Research Ethics Committee (Approval No. A17-12).

\section{BDNF and miR124a quantification after voluntary ethanol intake}

Rats were singly housed 10 days prior to the initiation of ethanol access. Fluid solutions were provided in cylindrical bottles equipped with regular steel drinking spouts. Rats were given two bottles one containing tap water, and the other containing either water $(n=8)$ or a solution of $5 \%$ ethanol in tap water $(\mathrm{v} / \mathrm{v} ; n=8)$ for 15 days. The placement order of the bottles was inverted daily to ensure that consumption levels were not due to a side preference.

Rats were killed $24 \mathrm{~h}$ after the final ethanol access by decapitation, and brain regions were dissected out and frozen in TRizol according to the manufacturer's instructions. The micro-dissection procedure was performed according to rat stereotaxic coordinates (Paxinos \& Watson, 1998), yielding lateral and medial subdivisions of the dorsal striatum [DLS and dorso-medial striatum (DMS), respectively]. The tissue was isolated via punch of a coronal slice $2.5 \mathrm{~mm}$ wide in the anterior-posterior direction, beginning immediately posterior to the prefrontal cortex (Supporting Information Fig. S1).

Total RNA was extracted from animal tissues using TRizol, and tested for purity $\left(A_{260 / 280}\right.$ ratios). Reverse transcription and real-time polymerase chain reaction (PCR) amplification was performed using Oligo-dT standard primers. Real-time PCR was performed with SYBR-Green. Reactions were performed in a $20-\mu \mathrm{L}$ final volume using $2 \mu \mathrm{L}$ of cDNA. The PCR protocol used consisted of a 45-s denaturation at $94{ }^{\circ} \mathrm{C}$, followed by 45 -s annealing and extension at $64{ }^{\circ} \mathrm{C}$ for 40 cycles. Glyceraldehyde-3-phosphate dehydrogenase $(\mathrm{GAPDH})$ was used as reference gene. The primers used were: miR124a: 5'-TCC GTG TTC ACA GCG.GAC-3' and 5'-CAT TCA CCG CGT GCC TTA-3' (Chandrasekar \& Dreyer, 2009); BDNF: 5'-GGT TCG AGA GGT CTG ACG AC-3' and 5'-CAA AGG CAC TTG ACT GCT GA-3'; GAPDH: 5'-ATG ACT CTA CCC ACG GCA AG-3' and 5'-CAT ACT CAG CAC CAG CAT CAC-3' (Bahi et al., 2008). 


\section{Lentiviral constructions and production}

The construction of the lentiviral vector (LV)-BDNF-expressing vector was performed as described previously (Bahi et al., 2008). Briefly, rat BDNF cDNA was amplified by PCR and cloned into pTK431 using BamHI and XhoI restriction sites. The construction of LV-miR124a- and LV-siR124a-expressing vectors was described in a previous study (Chandrasekar \& Dreyer, 2011). All constructions were confirmed by sequencing. For lentiviral production a triple transfection approach was used, as described previously (Bahi et al., 2005; Bahi \& Dreyer, 2008, 2012; Chandrasekar \& Dreyer, 2011). Briefly, the pTK431-expressing vectors were co-transfected into HEK293T cells together with the packaging $\mathrm{p} \Delta \mathrm{NRF}$ and the envelope pMDG-VSV-G plasmids. The viral particles were purified and concentrated using ultracentrifugation.

\section{Stereotaxic injection of LV-miR124a, LV-siR124a and $L V-B D N F$ in the dorsal striatum}

For stereotaxic surgery, rats were anesthetised with a ketamine/xylazine mixture $[100 \mathrm{mg} / \mathrm{kg}$ and $10 \mathrm{mg} / \mathrm{kg}$, respectively, intraperitoneally (i.p.)] and installed in a stereotaxic frame. Using a precision Hamilton micro-syringe with a $26 \mathrm{G}$ needle, rats were bilaterally infused with $2 \mu \mathrm{L}$ viral solution using the following coordinates: DLS (1.2 mm anterior to Bregma, $\pm 3.7 \mathrm{~mm}$ lateral to the medial suture, $4.5 \mathrm{~mm}$ ventral to the skull surface); medio-lateral striatum (MLS; $1.2 \mathrm{~mm}$ anterior to Bregma, $\pm 1.5 \mathrm{~mm}$ lateral to the medial suture, $4.5 \mathrm{~mm}$ ventral to the skull surface). All viral injections were performed according to the rat stereotaxic coordinates (Paxinos \& Watson, 1998). After surgery, animals were left in recovery for 7 days.

\section{Ethanol-induced CPP}

The place conditioning apparatus was a rectangular chamber that consisted of two conditioning compartments $(30 \times 30 \times 30 \mathrm{~cm})$ separated by a removable door $(10 \mathrm{~cm})$. One of the conditioning chambers had white walls and a stainless-steel large grid floor; the other had black walls and a narrow grid floor. Rats could access the entire apparatus when the guillotine door was removed (Bahi, 2012; Bahi \& Dreyer, 2012). The place conditioning procedure consisted of three phases: pre-conditioning, conditioning and post-conditioning tests, as previously described (Bahi \& Dreyer, 2012).

\section{Pre-conditioning}

The rats were injected with saline and placed between the two compartments with the guillotine doors removed, allowing free access to the two conditioning compartments for a 15 -min period. The time spent in each conditioning compartment was manually recorded. Most rats spent approximately $50 \%$ of the time in each chamber $(P>0.05)$. Therefore, conditioning was performed using an unbiased, balanced protocol.

After baseline assessment, the rats were stereotaxically injected with viral vectors as described above, and given a 7-day recovery period.

\section{Conditioning}

During conditioning, rats were injected i.p. with $0.5 \mathrm{~g} / \mathrm{kg}$ of ethanol (prepared from $20 \%$ ethanol solution dissolved in $0.9 \%$ isotonic saline $\mathrm{v} / \mathrm{v}$ ) once, and immediately confined to one conditioning compartment for $30 \mathrm{~min}$. On alternate schedules, rats received saline injections (volumes were calculated according to body weight) and were confined to the other compartment for $30 \mathrm{~min}$. The conditioning phase with ethanol and saline on alternative schedules (morning and afternoon) lasted for 5 days.

\section{Post-conditioning test}

Animals were injected with saline and immediately placed between the two compartments, with free access to both conditioning compartments for $15 \mathrm{~min}$. The time spent in each box was manually recorded. The preference for the ethanol-paired place was expressed as the mean difference between the duration spent in it during the pre- and post-conditioning tests.

\section{Two-bottle choice voluntary ethanol consumption and preference}

This procedure has been described previously (Bahi \& Dreyer, 2012). Briefly, two drinking bottles with $5 \%$ (v/v) alcohol or drinking tap water were available to the animals ad libitum during a 5day period. In order to avoid a possible side preference, the positions of the bottles were changed daily. The drinking bottles (g) and the body weight $(\mathrm{g})$ were weighed daily. The amount of consumed ethanol $(\mathrm{g})$ was calculated after every measurement and expressed per $1000 \mathrm{~g}$ of body weight $(1 \mathrm{~mL}=0.789 \mathrm{~g})$. The total fluid intake was worked out by calculating the volumes of water and ethanol consumed and adjusting to $1000 \mathrm{~g}$ of body weight.

Saccharin and quinine consumption and preference were measured in the same rats 7 days after measurement of ethanol drinking. Methods were as described for ethanol voluntary intake, and two concentrations of each tastant were evaluated (saccharin: 0.035 and $0.07 \%$; quinine: $0.015 \mathrm{~mm}$ and $0.03 \mathrm{~mm}$ ) in ascending order, consistent with our previous similar studies (Bahi \& Dreyer, 2012).

\section{BDNF and miR124a quantification following viral injection}

After completion of the quinine voluntary intake, rats were given access to two bottles - one containing tap water, and the other containing either water or a solution of $5 \%$ ethanol in tap water for 15 days. The placement order of the bottles was inverted daily to ensure that consumption levels were not due to a side preference. Rats were killed $24 \mathrm{~h}$ later by decapitation and tissue was processed as described previously.

\section{Determination of blood ethanol concentration (BEC)}

In brief, rats were given an i.p. injection with a $20 \%(\mathrm{v} / \mathrm{v})$ solution of ethanol ( $3 \mathrm{~g} / \mathrm{kg}$ of body weight) in isotonic sterile saline. Tail blood samples were collected in heparinised capillary tubes at 30, 60, 120 and $240 \mathrm{~min}$ following injection. The determinations of BEC expressed as $\mathrm{g} / \mathrm{dL}$ were made using the nicotinamide adenine dinucleotide (NAD)-NADH enzymatic assay, and concentrations were calculated using an ethanol standard curve.

\section{Statistical analysis}

For statistical comparisons, the software package SPSS (version 19.0) was used. All data were expressed as means \pm SEM. BDNF and miR124a mRNA expression were analysed using a two-way analysis of variance (ANOVA), with treatment and region as the between-subject factors. Ethanol-induced CPP behavior was analy- 
sed using a one-way ANOVA. Voluntary ethanol or tastant consumption and total volume intake were analysed using a two-way ANOva with repeated measures (virus group $\times$ ethanol/tastant concentration). BDNF and miR124a mRNA expression following viral injection were analysed using one-way ANOVAs. BEC data were analysed using one-way ANOVAS with repeated measures (virus groups were the between-subject factor). Post hoc individual mean comparisons were performed with the Bonferroni's test when $F$-values were significant. The level of statistical significance was set at $P<0.05$ at all times.

\section{Results}

\section{Expression of miR124a and BDNF in the dorsal striatum of rats following voluntary alcohol intake}

Figure 1 shows that miR124a and BDNF levels were affected following 15 days of voluntary alcohol intake in rats. In fact, and as revealed by a two-way ANOVA, miR124a expression was decreased about threefold in the DLS but not changed in the DMS (main effect of treatment $F_{1,28}=10.597, P=0.003$; main effect of region $F_{1,28}=5.921, P=0.022$ ). More importantly the interaction between the treatment and the region was significant $\left(F_{1,28}=9.001\right.$, $P=0.006$; Fig. 1A). Together these data indicate that chronic ethanol drinking results in the reduction of miR124a expression in the dorsal striatum, and that this effect is specific to the dorso-lateral region. To determine the potential role of miR124a in ethanol addiction, we studied the effect of miR124a on the expression of BDNF, a common signal molecule involved in basically all drugs of abuse, including alcohol (for review, see for example Corominas et al., 2007; Davis, 2008; McGinty et al., 2010; Autry \& Monteggia, 2012), in the rat dorsal striatum. As expected, 15-day alcohol intake significantly increased about twofold the expression of BDNF, as depicted in Fig. 1B; a two-way ANOva analysis revealed that, compared with the water-drinking group, BDNF expression was increased in the DLS of ethanol-exposed animals as indicated by a main effect of treatment $\left(F_{1,28}=5.492, P=0.026\right)$ and a main effect of region $\left(F_{1,28}=30.121, \quad P<0.001\right)$. More interestingly, the interaction between the two factors was significant $\left(F_{1,28}=7.464, P=0.011\right)$. These findings demonstrate that 2 weeks of ethanol intake upregulates dorso-lateral striatal BDNF.
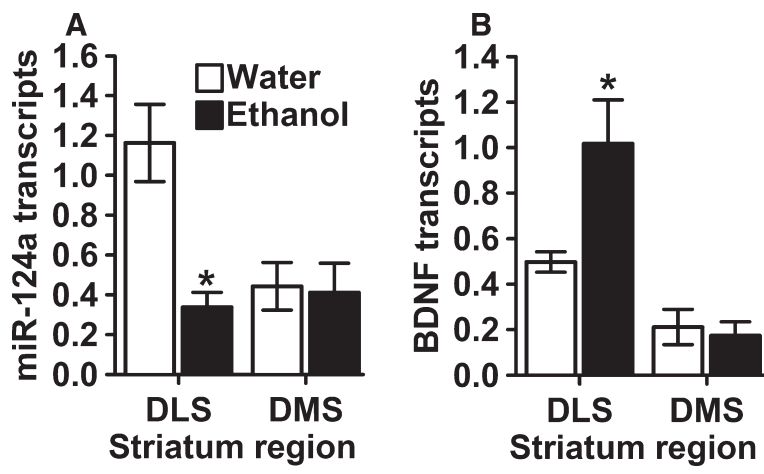

FIG. 1. Voluntary ethanol consumption induced changes in the miRNA124a and BDNF mRNA levels in the rat striatum. (A) miR124a and (B) BDNF mRNA levels were normalised against GAPDH in the corresponding samples. Histograms represent mean \pm SEM, $n=8, * P<0.05$ (two-way ANOvA, Bonferroni's post hoc test).
The effects of DLS LV-miR124a and LV-BDNF expression on ethanol-induced CPP

To test the effects of miR124a on ethanol-induced CPP, both gainof-function and loss-of-function approaches were used. In this experiment, LV-siR124a $(n=9)$ was used to knock down its expression, and a lentivirus-expressing miR124a (LV-miR124a; $n=8)$ was used to upregulate its expression in the DLS. LV-Mock- $(n=14)$ and LV-BDNF- $(n=12)$ expressing vectors were also used. During habituation to the CPP apparatus, no side preference was present in any of the groups, and there was no difference between the four groups $\left(F_{3,39}=0.528, P=0.666\right.$; data not shown). The effects of DLS lentiviral injections on ethanol-induced $(0.5 \mathrm{~g} / \mathrm{kg}) \mathrm{CPP}$ are shown in Fig. 2. One-way ANOva analysis has shown that the difference in time spent in the ethanol-paired box varied as a function of virus $\left(F_{3,39}=14.338, P<0.001\right)$. Post hoc evaluations revealed that rats injected with LV-BDNF or LV-siR124a showed about a threefold reduced CPP score compared with control rats (LV-Mock; $P=0.007$ and $P=0.009$, respectively). In addition, there was a slight difference between LV-Mock and LV-miR124a, but it did not reach significance $(P=0.075)$. More importantly, miR124a overexpression (LV-miR124a) enhanced about fivefold ethamol-induced CPP compared with LV-BDNF $(P<0.001)$ and LV-siR124a $(P<0.001)$. These findings suggested that miR124a is a critical regulator for BDNF in rat DLS. We hypothesise that miR124a might participate in the formation of alcohol conditioned reward by regulating the gene expression of BDNF.

\section{LV-siR124a in the DLS, but not LV-miR124a, attenuated voluntary alcohol intake in rats}

To determine levels of voluntary ethanol intake and preference following DLS viral injection of LV-Mock $(n=7)$, LV-miR124a $(n=8)$ and its silencer LV-siR124a $(n=9)$, we conducted a 5-day continuous-access two-bottle choice drinking test where animals could drink either water or a $5 \%$ ethanol solution. As depicted in Fig. 3A, one-way ANOvA repeated measure with virus as the between-subject factor showed no significant effect of time $\left(F_{4,84}=1.693, P=0.159\right)$. In contrast, there was a main significant effect of viral injection in the DLS on ethanol consumption

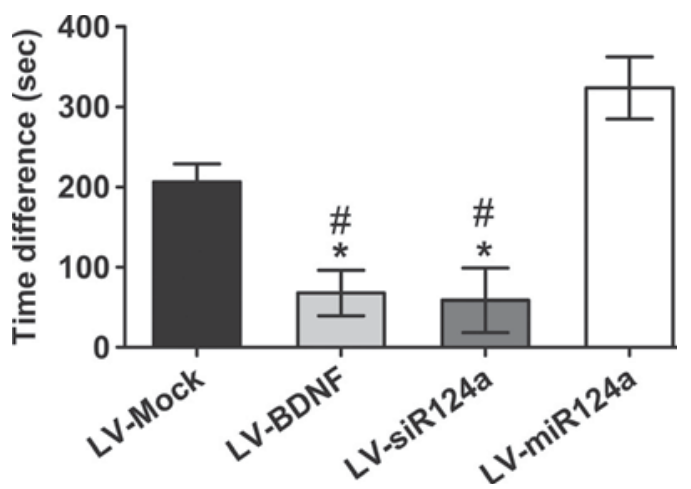

FIG. 2. Ethanol-induced CPP following viral injection in the DLS. Histograms show the mean \pm SEM of the time difference between post-conditioning and pre-conditioning tests that each group of rats spent in the ethanolpaired compartment. After baseline testing, animals were stereotaxically injected with LV-Mock, LV-BDNF, LV-siR124a or LV-miR124a. After recovery, animals were conditioned with either saline or ethanol (five conditioning sessions each), and then tested for their preference on day 11. $n=8-$ $14, * P<0.05$, compared with LV-Mock; ${ }^{\#} P<0.01$ compared with LVmiR124a (one-way ANOva, Bonferroni's post hoc test). 

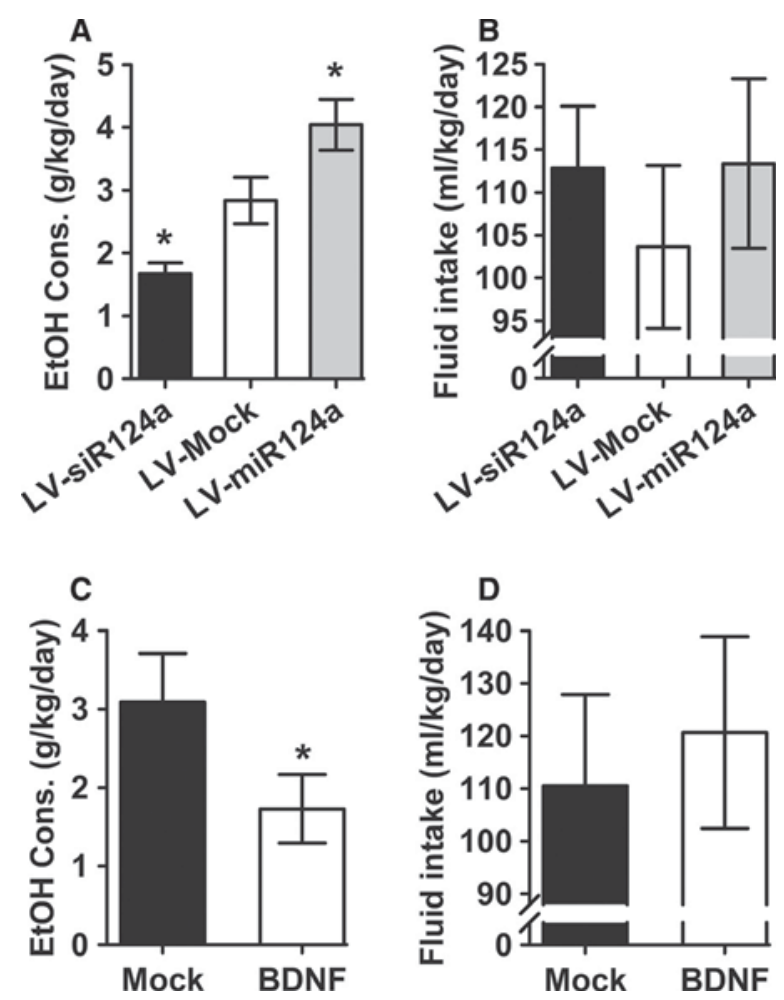

FIG. 3. Voluntary ethanol consumption and total fluid intake following viral injection in the DLS. Groups of Wistar rats were stereotaxically injected with LV-siR124a, LV-miR124a, LV-BDNF or LV-Mock, and given access to 5\% ethanol and water in a two-bottle choice drinking paradigm for 5 days. (A and C) Daily $\mathrm{g} / \mathrm{kg}$ ethanol consumption across the experiment. (B and D) The total fluid intake was the sum of the volume of ethanol solution and water consumed per $\mathrm{kg}$ of body weight per day. The data represent mean \pm SEM. The number of animals per group was $n=7-9$. $* P<0.05$ vs. LV-Mock (two-way ANOva, Bonferroni's post hoc test).

$\left(F_{2,21}=18.479, \quad P<0.001\right)$. Post hoc evaluations revealed that, compared with LV-Mock, LV-siR124a decreased (about 30\%, $P=0.027$ ) but LV-miR124a increased (about $25 \%, P=0.025$ ) voluntary ethanol intake. Interestingly, and as shown in Fig. 3B, daily total fluid intake did not differ between the three groups during the continuous-access two-bottle procedures as the virus main effect was not significant $\left(F_{2,21}=0.408, P=0.670\right)$. These findings suggest that the changed ethanol consumption by LV-siR124a- and LVmiR124a-injected rats was not caused by an overall change in total amount of fluid consumed.

\section{LV-BDNF in the DLS also attenuated voluntary alcohol intake in rats, similar to $L V$-siR124a}

The aim of this experiment was to determine whether the changes of expression of BDNF in the DLS can affect the voluntary alcohol drinking behaviors using the standard procedure in which the position of the bottles was alternated every day and drinking averaged across days. For this purpose, male rats were stereotaxically injected into the DLS with either LV-Mock- $(n=7)$ or LV-BDNF- $(n=12)$ expressing vectors, then given access to either $5 \%$ ethanol or tap water for five consecutive days. A significant difference in ethanol consumption was observed between LV-Mock- and LV-BDNFinjected rats $\left(F_{1,17}=16.671, P=0.001\right)$, with $\mathrm{LV}-\mathrm{BDNF}$ rats displaying a 1.7-fold decrease in ethanol intake when compared with controls (Fig. 3C). There was no significant effect of days of exposure to ethanol on alcohol intake $\left(F_{4,68}=1.422, P=0.236\right)$. As depicted in Fig. 3D, the one-way anova with repeated measure indicated a non-significant DLS viral injection effect on total fluid intake $\left(F_{1,17}=0.892, P=0.358\right)$. Thus, LV-Mock and LV-BDNF showed similar liquid consumption.

\section{LV-siR124a, LV-miR124a and LV-BDNF in the DLS did not affect taste discrimination}

To determine whether differences in ethanol voluntary intake might reflect changes in taste preferences caused by LV-siR124a and LVmiR124a injection, drinking studies with saccharin and quinine were performed. As shown in Fig. 4A, there was a significant difference in saccharin consumption across the two concentrations groups $\left[\left(F_{1,42}=91.167, P<0.001\right)\right.$ main effect of saccharin concentration $]$. This pattern was not altered by viral injection in the DLS $\left(F_{2,42}=0.209, \quad P=0.812\right)$. More importantly, the interaction between DLS viral injection and saccharin concentration was not significant $\left(F_{2,42}=0.127, P=0.881\right.$; two-way ANOvA with repeated measures, factors were: virus and saccharin concentration). Also, and as depicted in Fig. 4B, there were no differences between LVsiR124a-, LV-Mock- and LV-miR124a-injected rats in total fluid intake groups $\left[\left(F_{1,42}=6.872, P=0.012\right)\right.$ main effect of saccharin concentration; $\left(F_{2,42}=0.315, P=0.732\right)$ main effect of virus; $\left(F_{2,42}=0.020, P=0.980\right)$ main effect of saccharin concentration $\times$ virus interaction]. After 7 days of the saccharin intake study, the same rats were given access to quinine solution vs. water, the results are depicted in Fig. 4C. The consumption of quinine between the three viral-injected groups was not different $\left(F_{2,42}=0.225\right.$, $P=0.800)$, but rats consumed more quinine for the higher concentration $(0.03 \mathrm{~mm})$ compared with the lower concentration $(0.015 \mathrm{mM})$, as revealed by a main effect of quinine concentration $\left(F_{1,42}=19.845, P<0.001\right)$. No significant interaction between quinine concentration and viral injection in the DLS was found $\left(F_{2,42}=0.142, P=0.868\right)$. As for saccharin, the difference in total fluid intake in between the three groups was not significant $\left[\left(F_{1,42}=0.301, P=0.586\right)\right.$ main effect of quinine concentration; $\left(F_{2,42}=0.226, P=0.799\right)$ main effect of virus; $\left(F_{2,42}=0.521\right.$, $P=0.598)$ quinine concentration $\times$ virus interaction; Fig. 4D]. We compared the body weights of rats injected with LV-Mock, LVsiR124 and LV-miR124 at the end of the experiment, and the results have shown no main effect of virus $\left(F_{2,21}=2.309, P=0.124\right.$; data not shown).

To test the possibility that the differences in ethanol voluntary intake and preference for ethanol in LV-BDNF rats was due to differences in taste sensations rather than rewarding effects, we exposed rats to sweet solutions of saccharin and bitter solutions of quinine. As depicted in Fig. 4E, a two-way ANOVA with repeated measure revealed that there was no difference in the consumption for saccharin between LV-Mock and LV-BDNF $\left(F_{1,34}=0.263\right.$, $P=0.612)$. In contrast, there was a significant main effect of saccharin concentration $\left(F_{1,34}=72.023, P<0.001\right)$. More importantly, the interaction between viral injection in the DLS and saccharin concentration was not significant $\left(F_{1,34}=0.315, P=0.578\right)$. In addition, Fig. $4 \mathrm{~F}$ showed that there were no differences between $\mathrm{LV}$ Mock- and LV-BDNF-injected rats in total fluid intake groups $\left[\left(F_{1,34}=6.480, P=0.016\right)\right.$ main effect of saccharin concentration; $\left(F_{1,34}=0.103, P=0.750\right)$ main effect of virus; $\left(F_{1,34}=0.179\right.$, $P=0.675)$ saccharin concentration $\times$ virus interaction].

After 7 days of the saccharin intake study, the same rats were given access to quinine solution vs. water, the results are depicted 

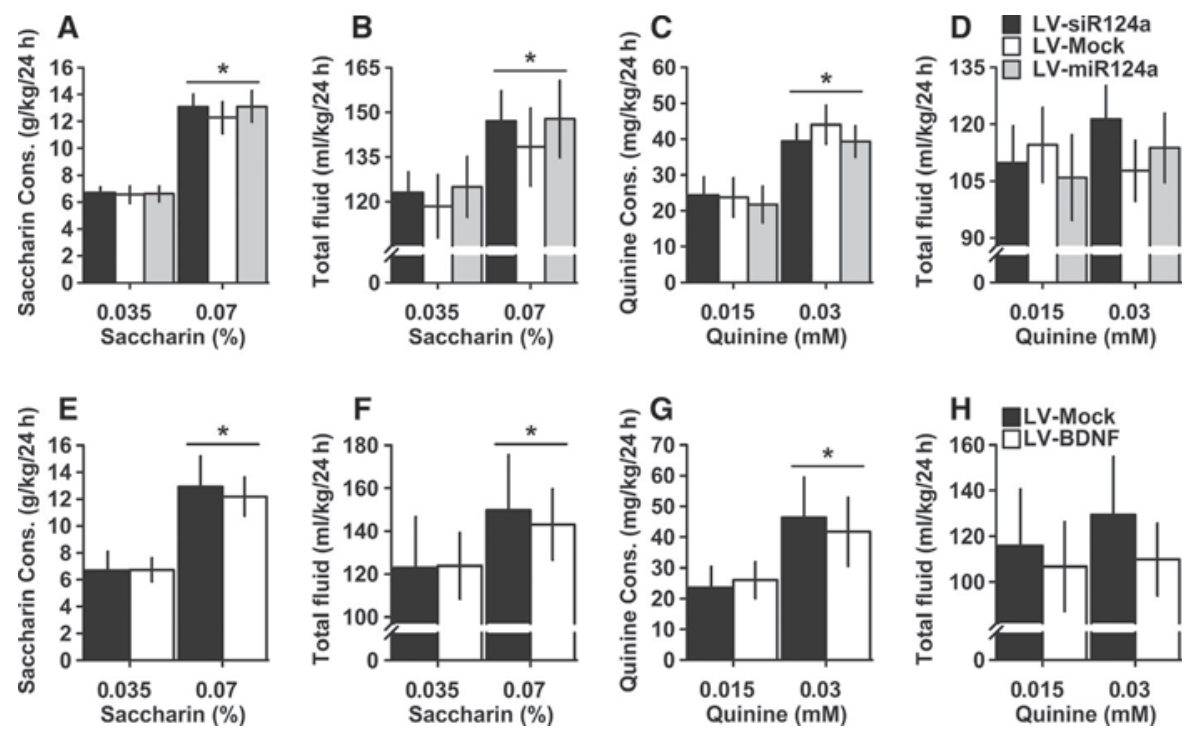

FIG. 4. Voluntary saccharin and quinine consumption, and total fluid intake following viral injection in the DLS. (A and E) Saccharin consumption; and (B and F) total fluid intake displayed by LV-siR124a, LV-miR124a, LV-BDNF and LV-Mock rats are indicated. Saccharin consumption was expressed as a gram of saccharin solution consumed per $\mathrm{kg}$ of body weight per day. The total fluid intake was the sum of the volume of the saccharin solution and water consumed per kg of body weight per day. (C and G) Quinine consumption; and (D and H) total fluid intake displayed by LV-siR124a, LV-miR124a, LV-BDNF and LVMock rats are shown. Quinine consumption was expressed as a mg of quinine solution consumed per kg of body weight per day. The total fluid intake was the sum of the volume of quinine solution and water consumed per $\mathrm{kg}$ of body weight per day. Data are presented as mean \pm SEM. $* P<0.05$, the difference between the indicated group (two-way ANOVA, Bonferroni's post hoc test).

in Fig. 4G. There was no difference $\left(F_{1,34}=1.136, P=0.294\right)$ between the two groups (LV-Mock and LV-BDNF) in terms of quinine consumption $(\mathrm{mg} / \mathrm{kg} / 24 \mathrm{~h})$. The ANOVA revealed a significant concentration effect $\left(F_{1,34}=20.507, \quad P<0.001\right)$, which reflected a general tendency towards higher intake of more concentrated quinine solutions. More importantly, the interaction between DLS viral injection and the quinine concentration was not significant $\left(F_{1,34}=0.061, P=0.807\right)$. However, the total fluid intake when the quinine solution was presented vs. water was similar between LV-Mock- and LV-BDNF-injected rats and was significant $\left[\left(F_{1,34}=0.838, P=0.366\right)\right.$ main effect of quinine concentration; $\quad\left(F_{1,34}=2.520, \quad P=0.122\right) \quad$ main effect of virus; $\left(F_{1,34}=0.322, P=0.574\right)$ interaction between quinine concentration and viral injection in the DLS; Fig. 4H]. Also, BDNF overexpression did not affect body weight $\left(F_{1,17}=1.743, P=0.204\right.$; data not shown).

\section{Expression of miR124a and BDNF in the dorsal striatum of rats following viral injections}

This experiment was designed to assess whether ethanol-induced upregulation of BDNF mRNA reported in Fig. 2 can be prevented by upregulation of miR124a. To test this hypothesis, LV-siR124a and LV-miR124a were infused into the DLS, and rats were given access to $5 \%$ ethanol for 15 days. As shown in Fig. 5 and as expected, one-way ANOVA revealed that LV-miR124a enhanced miR124a expression $\left(F_{2,13}=22.484, P<0.001\right)$. Post hoc evaluation indicated that, compared with LV-Mock, miRNA transcripts were upregulated with LV-miR124a $(P=0.002)$ and downregulated with LV-siR124a $(P=0.031)$. More importantly, BDNF mRNA was also regulated following miR124a modulation $\left(F_{2,13}=25.78\right.$, $P<0.001)$. In fact, and compared with LV-Mock, BDNF transcripts decreased following LV-miR124a injection (about threefold decrease, $P=0.001$ ) and increased upon LV-siR124a injection $(P=0.024)$.

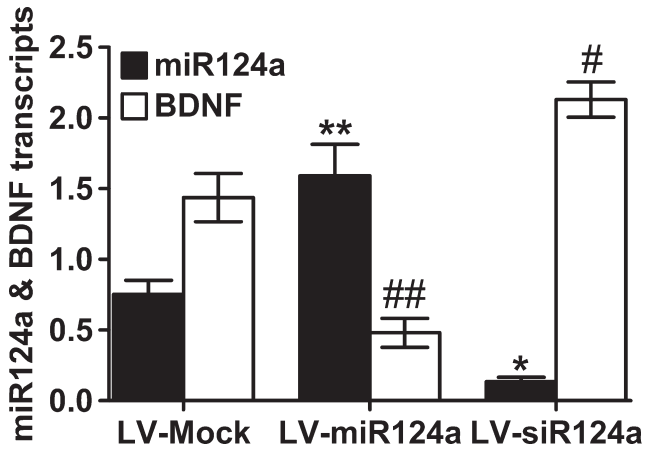

FIG. 5. Brain-derived neurotrophic factor (BDNF) and miR124a mRNA quantification following viral injection in the DLS and free access to $5 \%$ ethanol for 15 days. miR124a and BDNF quantification in LV-Mock-, LVsiR124a- and LV-miR124a-injected rats. Transcript levels were normalised against GAPDH $(n=7,4,5) . \quad * P<0.05, \quad * * P<0.005, \quad{ }^{\#} P<0.05$, \#\# $P<0.005$ (one-way Anova, Bonferroni's post hoc test).

In order to provide more convincing evidence for the direct regulation of the BDNF level by miR124A, rats were stereotaxically injected with LV-Mock, LV-miR124a or LV-siR124a in the DLS and, after recovery, BDNF mRNA was quantified after systemic ethanol injection $(2 \mathrm{~g} / \mathrm{kg}$, i.p.). As depicted in Fig. 6, there was a main effect of ethanol on BDNF mRNA expression $\left(F_{1,24}=22.128\right.$, $P<0.001)$. In fact, and compared with saline, LV-Mock-expressing rats displayed a 2.3-fold increase in BDNF mRNA upon ethanol administration. However, pre-miR124a injection abolished ethanolinduced BDNF mRNA increase as reflected by a main effect of virus injection $\left(F_{2,24}=9.536, P=0.001\right)$ and a significant interaction between the two factors $\left(F_{2,24}=10.534, P=0.001\right)$. More importantly, siR124a-injected rats displayed comparable BDNF mRNA expression as LV-Mock-expressing rats. Post hoc evaluations revealed a significant difference between Mock and miR124a 


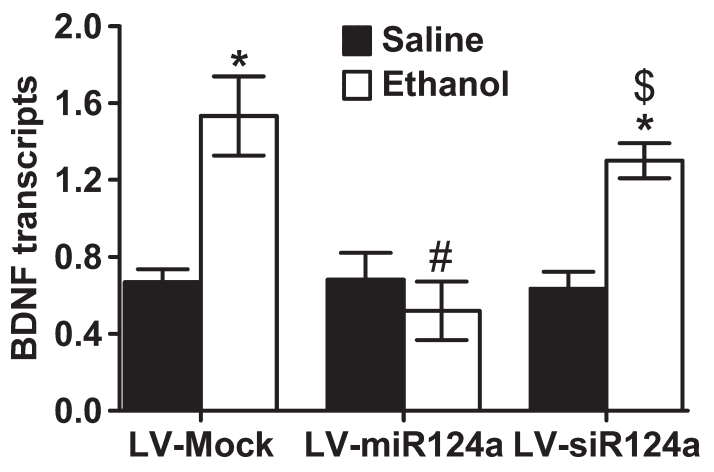

FIG. 6. Brain-derived neurotrophic factor (BDNF) mRNA quantification following viral infusion and passive i.p. injection of ethanol $(2 \mathrm{~g} / \mathrm{kg})$. BDNF quantification in LV-Mock-, LV-siR124a- and LV-miR124a-injected rats. Transcript levels were normalised against GAPDH $(n=5)$. $* P<0.01$ for ethanol vs. saline, ${ }^{\#} P<0.01$ for LV-miR124a vs. LV-Mock, ${ }^{\$} P<0.01$ for LV-siR124a vs. LV-miR124a (two-way ANOvA, Bonferroni's post hoc test).

$(P=0.001)$, but no difference between Mock and siR124a $(P=0.820)$. Taken together, these findings indicated that overexpression of miR124a indeed prevented alcohol-induced upregulation of BDNF.

\section{$B E C$ following viral injections of LV-miR124a, LV-siR124a and $L V-B D N F$}

Finally, the blood concentration of alcohol in LV-Mock-, LVmiR124a-, LV-siR124a- and LV-BDNF-injected rats was assessed. Results have shown that the viral vectors utilised in this study did not elicit their effects by altering ethanol pharmacodynamics (Supporting Information Fig. S2)

\section{Discussion}

In this study, we investigated ethanol-induced alteration of miR124a and BDNF mRNA expression in the dorsal striatum following voluntary ethanol intake. We found that ethanol consumption during 15 days resulted in a decrease in miR124a and an increase in BDNF expression in the DLS but not in the MLS. More importantly, we also demonstrated that lentiviral-mediated overexpression of miR124a in the DLS enhanced ethanol-induced CPP and voluntary alcohol intake in rats. In addition, knocking-down miR124a expression in the same brain region reduced ethanol-induced CPP and alcohol consumption. These last ethanol-induced behaviors were also observed with BDNF overexpression in the DLS. A control experiment revealed that tastants (saccharin and quinine) consumption was not affected following miR124a and BDNF modulation. In fact, both gain-of-function and loss-of-function approaches have demonstrated that miR124a was a strong regulator for the expression of BDNF in rat DLS. Moreover, the ethanol-mediated upregulation of BDNF was significantly blocked by overexpression of miR124a. Thus, miR124a indeed participated in alcohol-induced gene regulation in the rat brain. Based on these results, we hypothesise that continuous exposure to alcohol results in the induction of BDNF mRNA expression via miR124a downregulation.

miRNAs are small non-coding RNAs involved in the regulation of gene expression and protein translation. Along this line, we speculate that miRNAs may be involved in the regulation of drug-related genes in general (Hollander et al., 2010; Nudelman et al., 2010; Eipper-Mains et al., 2011) and ethanol in particular (Pietrzykowski et al., 2008; Miranda et al., 2010; Guo et al., 2012). To afford a direct support for ethanol-induced changes in miR124a expression, we assessed the expression pattern of miR124a in rat dorsal striatum after 15 days of exposure to voluntary alcohol intake. Our results clearly revealed that, compared with water, miR124a expression was downregulated in the DLS of alcohol-exposed rats. This result is in agreement with a previous study that reported that miR124a expression in the dorsal striatum of the rat brain was downregulated following cocaine exposure (Chandrasekar \& Dreyer, 2009). This downregulation was accompanied by a profound increase in mRNA levels of the miR124a direct target gene, BDNF (Chandrasekar \& Dreyer, 2009). In the same study, in silico computational analysis predicted that BDNF is a direct target gene of miR124a. Our results revealed that in rat DLS in vivo, alcohol-mediated increase of BDNF mRNA was attenuated by viral-mediated overexpression of miR124a.

Ethanol-induced modifications of gene expression in reward-associated brain regions are considered to contribute to long-lasting neuroadaptations and behavioral abnormalities (Lewohl et al., 2000; Kerns \& Miles, 2008; Piechota et al., 2010). In addition, extensive research has implicated BDNF neurotransmission in the regulation of responses to drug-related behavioral consequences in general (for review, see for example Corominas et al., 2007; Thomas et al., 2008; Dietz et al., 2009; Ghitza et al., 2010) and ethanol in particular (McGough et al., 2004; Davis, 2008; Bosse \& Mathews, 2011). How BDNF may contribute to the rewarding properties of alcohol is not yet known, but it has been shown that exposure to ethanol alters BDNF expression in several brain regions. In fact, a significant increase in BDNF mRNA was reported in the dorsal striatum upon voluntary ethanol consumption (McGough et al., 2004; Jeanblanc et al., 2009; Logrip et al., 2009).

The role of BDNF in alcohol addiction was assessed in rats using ethanol-induced CPP and voluntary access to two-bottle choice drinking paradigm. Our findings revealed that lentiviral-mediated overexpression of BDNF in rat DLS inhibited the development of ethanol-induced CPP and reduced voluntary alcohol consumption and preference. The current findings are in agreement with a previous report where McGough and co-workers have shown that BDNF \pm mice exhibit greater ethanol-induced place preference than control mice (McGough et al., 2004). In fact, genetic depletion of the levels of BDNF by $50 \%$ results in a significantly higher preference for the chamber associated to ethanol injection than that observed in wildtype control mice.

The importance of this BDNF mRNA regulatory mechanism is supported by several reports demonstrating a significant correlation between BDNF levels and alcohol intake. Thus, reduction in BDNF increases animal sensitivity for ethanol. In fact, it has been shown that after a deprivation period, both BDNF heterozygote mice consumed more ethanol than wild-type control male (McGough et al., 2004) and female mice (Hensler et al., 2003). Also, chronic heavy alcohol ingestion lowered BDNF levels in rats (Jung et al., 2011). More importantly and in humans, peripheral BDNF levels measured in alcohol-dependent patients and control subjects using an ELISA assay revealed that the BDNF level was lower in the alcohol-dependence group 'alcoholics' than in the normal controls (Joe et al., 2007; Huang et al., 2011). We hypothesise that ethanol exposure decreased the expression of miR124a and consequently increases the expression of BDNF in the dorsal striatum. Secreted BDNF, and through its receptor TrkB (Lamballe et al., 1991; Soppet et al., 1991), modulates the activity of neurotransmitter systems, especially dopamine and serotonin. Thus, BDNF signaling pathway activation was shown to induce expression of the dopamine D3 receptor (Jean- 
blanc et al., 2006), and increase serotonin synthesis and release (Mamounas et al., 2000). More importantly, it has been reported that activation of the dopamine D3 receptor negatively regulates ethanol intake (Thanos et al., 2005). Also, decreased serotonergic function was associated with increased alcohol preference and consumption in rodents (for review, see LeMarquand et al., 1994a, b). Together, these data support the theory that BDNF functions as a positive modulator of ethanol intake in both laboratory animals and humans, most probably through the dopamine D3 receptor and the serotoninergic system by blocking the progression to addiction.

In addition, we found that striatal miR124a overexpression exacerbated the stimulatory effect of ethanol. Moreover, inhibition of striatal miR124a signaling using a lentiviral-mediated silencer expression 'rescued' the decreased alcohol intake and ethanolinduced CPP intake seen in BDNF-overexpressing rats. Therefore, we hypothesise that miR124a controls alcohol intake, and may influence vulnerability to ethanol addiction, by regulating the stimulatory effects of the drug on striatal BDNF expression. Whatever the underlying mechanisms, our data demonstrate that siR124a and miR124a exert opposite effects on striatal BDNF expression levels, and suggest that the balance between these two factors may play a crucial role in determining vulnerability to ethanol-rewarding properties and addiction.

In summary, the expression profile of miR124a in rat dorsal striatum after ethanol exposure was investigated. The results suggested that miR124a was downregulated following alcohol. Consequently, we hypothesise that miR124a is an important regulator of alcohol addiction via its direct target gene BDNF. The current findings focus attention on the fact that miR124a enables fine-tuning of BDNFinduced transcriptional neuroplastic responses to drugs of abuse. Indeed, the highly coordinated action of miR124a on BDNF, and perhaps on many other addiction-related genes, suggests that miR124a may be a key focal point in controlling ethanol-induced striatal neuroplasticity and vulnerability to alcohol addiction.

\section{Supporting Information}

Additional supporting information can be found in the online version of this article:

Fig. S1. Histological representation of dissection placements in the dorso-lateral "DLS" (A) and medio-lateral "MLS" striatum (B).

Fig. S2. Mean blood ethanol concentrations following viral injection. (A) Mean BEC of LV-siR124a, LV-Mock and LV-miR124a injected rats $(n=4,3,4)$. (B) Mean BEC of LV-Mock and LVBDNF -injected rats $(n=3 \& 6)$. For all groups, viral vectors were injected into the DLS and BEC was assessed following a single injection with ethanol ( $3 \mathrm{~g} / \mathrm{kg}$ body weight, i.p.). Blood was collected from the tail vein at various times after injection and analysed to determine the ethanol concentration. Values represent the mean \pm SEM. $* P<0.000$, the difference between 0.5 - and 4 -h (repeated measures one-way ANova, Bonferroni's post hoc test).

\section{Disclosure/Conflict of Interest}

The authors report no conflicts of interest.

\section{Acknowledgements}

The authors would like to acknowledge Dr Vijay Chandrasekar for helpful discussions, and the design and preparation of LV-miR124a and LV-si124a. The authors are grateful also to Mrs Christine Deforel-Poncet, Mr Mohamed Elwasila and Mr Mohamed Shafiullah for their technical assistance. A.B. is receiving funds from the United Arab Emirates University (salary and research funding). J.L.D. was supported by Swiss National Science Foundation (Grants no. 3100-059350, 3100AO-100686 and 31003A-116492). The funders had no role in study design, data collection and analysis, decision to publish, or preparation of the manuscript.

\section{Abbreviations}

BDNF, brain-derived neurotrophic factor; BEC, blood ethanol concentration; CPP, conditioned-place preference; DLS, dorso-lateral striatum; DMS, dorso-medial striatum; GAPDH, glyceraldehyde-3-phosphate dehydrogenase; i.p., intraperitoneally; LV, lentiviral vector; miRNA, microRNA; MLS, medio-lateral striatum; PCR, polymerase chain reaction; TrkB, tropomyosinrelated kinase $\mathrm{B}$.

\section{References}

Autry, A.E. \& Monteggia, L.M. (2012) Brain-derived neurotrophic factor and neuropsychiatric disorders. Pharmacol. Rev., 64, 238-258.

Bahi, A. (2012) The selective metabotropic glutamate receptor 7 allosteric agonist AMN082 prevents reinstatement of extinguished ethanol-induced conditioned place preference in mice. Pharmacol. Biochem. Be., 101, 193200.

Bahi, A. \& Dreyer, J.L. (2008) Overexpression of plasminogen activators in the nucleus accumbens enhances cocaine-, amphetamine- and morphineinduced reward and behavioral sensitization. Genes Brain Behav., 7, 244 256.

Bahi, A. \& Dreyer, J.L. (2012) Involvement of nucleus accumbens dopamine D1 receptors in ethanol drinking, ethanol-induced conditioned place preference, and ethanol-induced psychomotor sensitization in mice. Psychopharmacology, 222, 141-153.

Bahi, A., Boyer, F., Bussard, G. \& Dreyer, J.L. (2005) Silencing dopamine D3-receptors in the nucleus accumbens shell in vivo induces changes in cocaine-induced hyperlocomotion. Eur. J. Neurosci., 21, 3415-3426.

Bahi, A., Boyer, F., Chandrasekar, V. \& Dreyer, J.L. (2008) Role of accumbens BDNF and TrkB in cocaine-induced psychomotor sensitization, conditioned-place preference, and reinstatement in rats. Psychopharmacology, 199, 169-182.

Bosse, K.E. \& Mathews, T.A. (2011) Ethanol-induced increases in extracellular dopamine are blunted in brain-derived neurotrophic factor heterozygous mice. Neurosci. Lett., 489, 172-176.

Briand, L.A. \& Blendy, J.A. (2010) Molecular and genetic substrates linking stress and addiction. Brain Res., 1314, 219-234.

Cao, X., Yeo, G., Muotri, A.R., Kuwabara, T. \& Gage, F.H. (2006) Noncoding RNAs in the mammalian central nervous system. Annu. Rev. Neurosci., 29, 77-103.

Cao, X., Pfaff, S.L. \& Gage, F.H. (2007) A functional study of miR-124 in the developing neural tube. Gene. Dev., 21, 531-536.

Caputo, V., Sinibaldi, L., Fiorentino, A., Parisi, C., Catalanotto, C., Pasini, A., Cogoni, C. \& Pizzuti, A. (2011) Brain derived neurotrophic factor (BDNF) expression is regulated by microRNAs miR-26a and miR-26b allele-specific binding. PLoS One, 6, e28656.

Chandrasekar, V. \& Dreyer, J.L. (2009) microRNAs miR-124, let-7d and miR-181a regulate cocaine-induced plasticity. Mol. Cell. Neurosci., 42, $350-362$.

Chandrasekar, V. \& Dreyer, J.L. (2011) Regulation of MiR-124, Let-7d, and MiR-181a in the accumbens affects the expression, extinction, and reinstatement of cocaine-induced conditioned place preference. Neuropsychopharmacol., 36, 1149-1164.

Cheng, L.C., Pastrana, E., Tavazoie, M. \& Doetsch, F. (2009) miR-124 regulates adult neurogenesis in the subventricular zone stem cell niche. Nat. Neurosci., 12, 399-408.

Corominas, M., Roncero, C., Ribases, M., Castells, X. \& Casas, M. (2007) Brain-derived neurotrophic factor and its intracellular signaling pathways in cocaine addiction. Neuropsychobiology, 55, 2-13.

Cui, Q., Yu, Z., Pan, Y., Purisima, E.O. \& Wang, E. (2007) MicroRNAs preferentially target the genes with high transcriptional regulation complexity. Biochem. Bioph. Res. Co., 352, 733-738.

Davis, M.I. (2008) Ethanol-BDNF interactions: still more questions than answers. Pharmacol. Therapeut., 118, 36-57.

Dietz, D.M., Dietz, K.C., Nestler, E.J. \& Russo, S.J. (2009) Molecular mechanisms of psychostimulant-induced structural plasticity. Pharmacopsychiatry, 42(Suppl 1), S69-78. 
Dreyer, J.L. (2010) New insights into the roles of microRNAs in drug addiction and neuroplasticity. Genome Med., 2, 92.

Eipper-Mains, J.E., Kiraly, D.D., Palakodeti, D., Mains, R.E., Eipper, B.A \& Graveley, B.R. (2011) microRNA-Seq reveals cocaine-regulated expression of striatal microRNAs. RNA, 17, 1529-1543.

Everitt, B.J. \& Robbins, T.W. (2005) Neural systems of reinforcement for drug addiction: from actions to habits to compulsion. Nat. Neurosci., 8, 1481-1489.

Everitt, B.J. \& Robbins, T.W. (2013) From the ventral to the dorsal striatum: devolving views of their roles in drug addiction. Neurosci. Biobehav. R., 7634, 00046-00048.

Everitt, B.J., Belin, D., Economidou, D., Pelloux, Y., Dalley, J.W. \& Robbins, T.W. (2008) Review. Neural mechanisms underlying the vulnerability to develop compulsive drug-seeking habits and addiction. Philos. T. Roy. Soc. B., 363, 3125-3135.

Fang, M., Wang, J., Zhang, X., Geng, Y., Hu, Z., Rudd, J.A., Ling, S., Chen, W. \& Han, S. (2012) The miR-124 regulates the expression of BACE1/beta-secretase correlated with cell death in Alzheimer's disease Toxicol. Lett., 209, 94-105.

Fasanaro, P., Greco, S., Lorenzi, M., Pescatori, M., Brioschi, M., Kulshreshtha, R., Banfi, C., Stubbs, A., Calin, G.A., Ivan, M., Capogrossi, M.C. \& Martelli, F. (2009) An integrated approach for experimental target identification of hypoxia-induced miR-210. J. Biol. Chem., 284, 35134-35143.

Filipowicz, W., Bhattacharyya, S.N. \& Sonenberg, N. (2008) Mechanisms of post-transcriptional regulation by microRNAs: are the answers in sight? Nat. Rev. Genet., 9, 102-114.

Franke, K., Otto, W., Johannes, S., Baumgart, J., Nitsch, R. \& Schumacher, S. (2012) miR-124-regulated RhoG reduces neuronal process complexity via ELMO/Dock180/Rac1 and Cdc42 signalling. EMBO J., 31, 2908-2921.

Friedman, L.M., Dror, A.A., Mor, E., Tenne, T., Toren, G., Satoh, T., Biesemeier, D.J., Shomron, N., Fekete, D.M., Hornstein, E. \& Avraham, K.B (2009) MicroRNAs are essential for development and function of inner ear hair cells in vertebrates. Proc. Natl. Acad. Sci. USA, 106, 7915-7920.

Gerdeman, G.L., Partridge, J.G., Lupica, C.R. \& Lovinger, D.M. (2003) It could be habit forming: drugs of abuse and striatal synaptic plasticity. Trends Neurosci., 26, 184-192.

Ghitza, U.E., Zhai, H., Wu, P., Airavaara, M., Shaham, Y. \& Lu, L. (2010) Role of BDNF and GDNF in drug reward and relapse: a review. Neurosci. Biobehav. R., 35, 157-171.

Guo, Y., Chen, Y., Carreon, S. \& Qiang, M. (2012) Chronic intermittent ethanol exposure and its removal induce a different miRNA expression pattern in primary cortical neuronal cultures. Alcohol. Clin. Exp. Res., 36, 1058-1066.

Hensler, J.G., Ladenheim, E.E. \& Lyons, W.E. (2003) Ethanol consumption and serotonin-1A (5-HT1A) receptor function in heterozygous BDNF $( \pm)$ mice. J. Neurochem., 85, 1139-1147.

Hollander, J.A., Im, H.I., Amelio, A.L., Kocerha, J., Bali, P., Lu, Q., Willoughby, D., Wahlestedt, C., Conkright, M.D. \& Kenny, P.J. (2010) Striatal microRNA controls cocaine intake through CREB signalling. Nature, 466, 197-202.

Huang, M.C., Chen, C.H., Liu, H.C., Chen, C.C., Ho, C.C. \& Leu, S.J. (2011) Differential patterns of serum brain-derived neurotrophic factor levels in alcoholic patients with and without delirium tremens during acute withdrawal. Alcohol. Clin. Exp. Res., 35, 126-131.

Impey, S., Davare, M., Lesiak, A., Fortin, D., Ando, H., Varlamova, O., Obrietan, K., Soderling, T.R., Goodman, R.H. \& Wayman, G.A. (2010) An activity-induced microRNA controls dendritic spine formation by regulating Rac1-PAK signaling. Mol. Cell. Neurosci., 43, 146-156.

Jeanblanc, J., He, D.Y., McGough, N.N., Logrip, M.L., Phamluong, K., Janak, P.H. \& Ron, D. (2006) The dopamine D3 receptor is part of a homeostatic pathway regulating ethanol consumption. J. Neurosci., 26, 1457-1464

Jeanblanc, J., He, D.Y., Carnicella, S., Kharazia, V., Janak, P.H. \& Ron, D (2009) Endogenous BDNF in the dorsolateral striatum gates alcohol drinking. J. Neurosci., 29, 13494-13502.

Joe, K.H., Kim, Y.K., Kim, T.S., Roh, S.W., Choi, S.W., Kim, Y.B., Lee, H.J. \& Kim, D.J. (2007) Decreased plasma brain-derived neurotrophic factor levels in patients with alcohol dependence. Alcohol. Clin. Exp. Res., 31, 1833-1838.

Jonkman, S. \& Kenny, P.J. (2013) Molecular, cellular, and structural mechanisms of cocaine addiction: a key role for microRNAs. Neuropsychopharmacol., 38, 198-211.

Jung, K.I., Ju, A., Lee, H.M., Lee, S.S., Song, C.H., Won, W.Y., Jeong, J.S., Hong, O.K., Kim, J.H. \& Kim, D.J. (2011) Chronic ethanol ingestion, type 2 diabetes mellitus, and brain-derived neurotrophic factor (BDNF) in rats. Neurosci. Lett., 487, 149-152.
Karpova, N.N., Pickenhagen, A., Lindholm, J., Tiraboschi, E., Kulesskaya, N., Agustsdottir, A., Antila, H., Popova, D., Akamine, Y., Bahi, A., Sullivan, R., Hen, R., Drew, L.J. \& Castren, E. (2011) Fear erasure in mice requires synergy between antidepressant drugs and extinction training. Science, 334, 1731-1734.

Kawahara, H., Imai, T. \& Okano, H. (2012) MicroRNAs in neural stem cells and neurogenesis. Front. Neurosci., 6, 30.

Kerns, R.T. \& Miles, M.F. (2008) Microarray analysis of ethanol-induced changes in gene expression. Methods Mol. Biol., 447, 395-410.

Kim, J., Inoue, K., Ishii, J., Vanti, W.B., Voronov, S.V., Murchison, E., Hannon, G. \& Abeliovich, A. (2007) A MicroRNA feedback circuit in midbrain dopamine neurons. Science, 317, 1220-1224.

Kuss, A.W. \& Chen, W. (2008) MicroRNAs in brain function and disease. Curr. Neurol. Neurosci., 8, 190-197.

Lamballe, F., Klein, R. \& Barbacid, M. (1991) The trk family of oncogenes and neurotrophin receptors. Princess Takamatsu S., 22, 153-170.

LeMarquand, D., Pihl, R.O. \& Benkelfat, C. (1994a) Serotonin and alcohol intake, abuse, and dependence: clinical evidence. Biol. Psychiat., 36, 326337

LeMarquand, D., Pihl, R.O. \& Benkelfat, C. (1994b) Serotonin and alcohol intake, abuse, and dependence: findings of animal studies. Biol. Psychiat., 36, 395-421.

Lewis, B.P., Shih, I.H., Jones-Rhoades, M.W., Bartel, D.P. \& Burge, C.B (2003) Prediction of mammalian microRNA targets. Cell, 115, 787-798.

Lewohl, J.M., Wang, L., Miles, M.F., Zhang, L., Dodd, P.R. \& Harris, R.A. (2000) Gene expression in human alcoholism: microarray analysis of frontal cortex. Alcohol. Clin. Exp. Res., 24, 1873-1882.

Logrip, M.L., Janak, P.H. \& Ron, D. (2009) Escalating ethanol intake is associated with altered corticostriatal BDNF expression. J. Neurochem., 109, 1459-1468.

Makeyev, E.V., Zhang, J., Carrasco, M.A. \& Maniatis, T. (2007) The MicroRNA miR-124 promotes neuronal differentiation by triggering brain-specific alternative pre-mRNA splicing. Mol. Cell, 27, 435-448.

Mamounas, L.A., Altar, C.A., Blue, M.E., Kaplan, D.R., Tessarollo, L. \& Lyons, W.E. (2000) BDNF promotes the regenerative sprouting, but not survival, of injured serotonergic axons in the adult rat brain. J. Neurosci., 20, 771-782.

McGinty, J.F., Whitfield, T.W. Jr. \& Berglind, W.J. (2010) Brain-derived neurotrophic factor and cocaine addiction. Brain Res., 1314, 183-193.

McGough, N.N., He, D.Y., Logrip, M.L., Jeanblanc, J., Phamluong, K., Luong, K., Kharazia, V., Janak, P.H. \& Ron, D. (2004) RACK1 and brainderived neurotrophic factor: a homeostatic pathway that regulates alcohol addiction. J. Neurosci., 24, 10542-10552.

Mellios, N., Huang, H.S., Grigorenko, A., Rogaev, E. \& Akbarian, S. (2008) A set of differentially expressed miRNAs, including miR-30a-5p, act as post-transcriptional inhibitors of BDNF in prefrontal cortex. Hum. Mol. Genet., 17, 3030-3042.

Miranda, R.C., Pietrzykowski, A.Z., Tang, Y., Sathyan, P., Mayfield, D., Keshavarzian, A., Sampson, W. \& Hereld, D. (2010) MicroRNAs: master regulators of ethanol abuse and toxicity? Alcohol. Clin. Exp. Res., 34, 575-587.

Nestler, E.J. \& Carlezon, W.A. Jr. (2006) The mesolimbic dopamine reward circuit in depression. Biol. Psychiat., 59, 1151-1159.

Nudelman, A.S., DiRocco, D.P., Lambert, T.J., Garelick, M.G., Le, J., Nathanson, N.M. \& Storm, D.R. (2010) Neuronal activity rapidly induces transcription of the CREB-regulated microRNA-132, in vivo. Hippocampus, 20, 492-498.

Paxinos, G. \& Watson, C. (1998) The Rat Brain in Stereotaxic Coordinates. Academic Press, San Diego.

Piechota, M., Korostynski, M., Solecki, W., Gieryk, A., Slezak, M., Bilecki, W., Ziolkowska, B., Kostrzewa, E., Cymerman, I., Swiech, L., Jaworski, J. \& Przewlocki, R. (2010) The dissection of transcriptional modules regulated by various drugs of abuse in the mouse striatum. Genome Biol., 11, R48.

Pietrzykowski, A.Z., Friesen, R.M., Martin, G.E., Puig, S.I., Nowak, C.L. Wynne, P.M., Siegelmann, H.T. \& Treistman, S.N. (2008) Posttranscriptional regulation of BK channel splice variant stability by miR-9 underlies neuroadaptation to alcohol. Neuron, 59, 274-287.

Pruunsild, P., Kazantseva, A., Aid, T., Palm, K. \& Timmusk, T. (2007) Dissecting the human BDNF locus: bidirectional transcription, complex splicing, and multiple promoters. Genomics, 90, 397-406.

Qi, L., Hu, Y., Zhan, Y., Wang, J., Wang, B.B., Xia, H.F. \& Ma, X. (2012) A SNP site in pri-miR-124 changes mature miR-124 expression but no contribution to Alzheimer's disease in a Mongolian population. Neurosci. Lett., 515, 1-6. 
Sato, F., Tsuchiya, S., Meltzer, S.J. \& Shimizu, K. (2011) MicroRNAs and epigenetics. FEBS J., 278, 1598-1609.

Silber, J., Lim, D.A., Petritsch, C., Persson, A.I., Maunakea, A.K., Yu, M., Vandenberg, S.R., Ginzinger, D.G., James, C.D., Costello, J.F., Bergers, G., Weiss, W.A., Alvarez-Buylla, A. \& Hodgson, J.G. (2008) miR-124 and miR-137 inhibit proliferation of glioblastoma multiforme cells and induce differentiation of brain tumor stem cells. BMC Med., 6, 14 .

Silber, J., James, C.D. \& Hodgson, J.G. (2009) microRNAs in gliomas: small regulators of a big problem. Neuromol. Med., 11, 208-222.

Simerly, R. (2006) Feeding signals and drugs meet in the midbrain. Nat. Med., 12, 1244-1246.

Soppet, D., Escandon, E., Maragos, J., Middlemas, D.S., Reid, S.W., Blair J., Burton, L.E., Stanton, B.R., Kaplan, D.R., Hunter, T., Nikolics, K. \& Parada, L.F. (1991) The neurotrophic factors brain-derived neurotrophic factor and neurotrophin-3 are ligands for the trkB tyrosine kinase receptor. Cell, 65, 895-903.

Stuber, G.D., Hopf, F.W., Tye, K.M., Chen, B.T. \& Bonci, A. (2010) Neuroplastic alterations in the limbic system following cocaine or alcohol exposure. Curr. Top. Behav. Neurosci., 3, 3-27.

Sun, K., Westholm, J.O., Tsurudome, K., Hagen, J.W., Lu, Y., Kohwi, M., Betel, D., Gao, F.B., Haghighi, A.P., Doe, C.Q. \& Lai, E.C. (2012) Neurophysiological defects and neuronal gene deregulation in Drosophila mir124 mutants. PLoS Genet., 8, e1002515.

Thanos, P.K., Katana, J.M., Ashby, C.R. Jr., Michaelides, M., Gardner, E.L., Heidbreder, C.A. \& Volkow, N.D. (2005) The selective dopamine D3 receptor antagonist SB-277011-A attenuates ethanol consumption in ethanol preferring (P) and non-preferring (NP) rats. Pharmacol. Biochem. Be., 81, 190-197.

Thomas, M.J., Kalivas, P.W. \& Shaham, Y. (2008) Neuroplasticity in the mesolimbic dopamine system and cocaine addiction. Brit. J. Pharmacol., 154, 327-342.
Thomson, J.M., Newman, M., Parker, J.S., Morin-Kensicki, E.M., Wright, T. \& Hammond, S.M. (2006) Extensive post-transcriptional regulation of microRNAs and its implications for cancer. Gene. Dev., 20, 2202-2207.

Tricomi, E., Balleine, B.W. \& O'Doherty, J.P. (2009) A specific role for posterior dorsolateral striatum in human habit learning. Eur. J. Neurosci., 29, $2225-2232$.

Visvanathan, J., Lee, S., Lee, B., Lee, J.W. \& Lee, S.K. (2007) The microRNA miR-124 antagonizes the anti-neural REST/SCP1 pathway during embryonic CNS development. Gene. Dev., 21, 744-749.

Xia, H., Cheung, W.K., Ng, S.S., Jiang, X., Jiang, S., Sze, J., Leung, G.K., Lu, G., Chan, D.T., Bian, X.W., Kung, H.F., Poon, W.S. \& Lin, M.C. (2012a) Loss of brain-enriched miR-124 microRNA enhances stem-like traits and invasiveness of glioma cells. J. Biol. Chem., 287, 9962-9971.

Xia, J., Wu, Z., Yu, C., He, W., Zheng, H., He, Y., Jian, W., Chen, L., Zhang, L. \& Li, W. (2012b) MiR-124 inhibits cell proliferation in gastric cancer through downregulation of SPHK1. J. Pathol., 227, 470-480.

Yin, H.H. \& Knowlton, B.J. (2006) The role of the basal ganglia in habit formation. Nat. Rev. Neurosci., 7, 464-476.

Yin, H.H., Knowlton, B.J. \& Balleine, B.W. (2004) Lesions of dorsolateral striatum preserve outcome expectancy but disrupt habit formation in instrumental learning. Eur. J. Neurosci., 19, 181-189.

Yin, H.H., Knowlton, B.J. \& Balleine, B.W. (2006) Inactivation of dorsolateral striatum enhances sensitivity to changes in the action-outcome contingency in instrumental conditioning. Behav. Brain Res., 166, 189-196.

Zheng, F., Liao, Y.J., Cai, M.Y., Liu, Y.H., Liu, T.H., Chen, S.P., Bian, X.W., Guan, X.Y., Lin, M.C., Zeng, Y.X., Kung, H.F. \& Xie, D. (2012) The putative tumour suppressor microRNA-124 modulates hepatocellular carcinoma cell aggressiveness by repressing ROCK2 and EZH2. Gut, 61, 278-289. 